\title{
Versatile Controllability over Cell Switching for Speedy Users in LTE HetNets
}

\author{
Mohammad T. KAWSER, Mohammad R. ISLAM, Muhammad R. RAHIM, Muhammad A. MASUD
}

Dept. of Electrical and Electronic Engineering, Islamic University of Technology, Board Bazar, Gazipur-1704, Bangladesh

mkawser@hotmail.com,rakibultowhid@yahoo.com,ridwan351990@gmail.com, atique76i@gmail.com

Submitted April 30, 2016 / Accepted November 17, 2016

\begin{abstract}
The heterogeneous networks (HetNets) are regarded as a promising solution in LTE-Advanced for ubiquitous and cost effective broadband user experience. But there are challenges to support seamless mobility in HetNets, especially, when the user speed is high. In this paper, we investigate these challenges and study the scopes to address them for the improvement of cell edge performance. The study indicates the requirement of enhanced and versatile controllability over adaptation of cell switching parameters that simultaneously depends on variation in user speeds, traffic loads, street patterns, types of cells involved in switching, and so forth. We propose a scheme to scale cell switching parameters that incorporates Doppler spread estimation and adapts smoothly to various changes. Both the eNodeB and the UE participate in a versatile control over the scaling. Limited simulations have been performed to partially reflect the outcome of the proposed scheme.
\end{abstract}

\section{Keywords}

LTE, HetNets, cell reselection, handover, speedy users

\section{Introduction}

Long term evolution (LTE), and its later version LTEAdvanced, are the latest steps in the advancing series of cellular communication technologies. The heterogeneous networks (HetNets) are introduced in LTE-Advanced to enhance coverage and capacity greatly. A HetNet consists of regular macro cells typically transmitting at high power level, overlaid with low power small cells such as pico cell, femto cell, remote radio head (RRH), and relay node (RN) [1]. In this paper, in addition to the macro cell, the HetNet is considered to have only pico cells and femto cells and they both are referred to as small cells. The small cells offload traffic from the macro cell and offer extension of the reach of coverage. They improve the conditions in coverage holes providing higher data rates at cell edge or in hotspots. The small cells have smaller base stations with lower antenna gain compared to macro cells and so, their site acquisition can be simpler. However, there are some challenges that need to be addressed in HetNets.
The serving cell is updated automatically with the movement of the user to support user mobility in LTE. In the RRC_IDLE state, the cell reselection procedure is performed to change the cell on which the user equipment (UE) is camped. In the RRC_CONNECTED state, handover is performed to change the serving cell. The decision for the change of cell depends on the relative radio link quality between what are experienced by the UE from the currently serving cell and from the neighbor cells. Because of the time varying nature of the radio signals, it is possible that what appears to be an increase or decrease of the received signal strength is actually a transient fluctuation. Thus, the decision for cell switching must allow the target cell to remain better than the serving cell by a sufficient margin continuously for a sufficient period. Otherwise, there is a possibility of switching back and forth between cells unnecessarily, which is known as ping-pong events. The ping-pong events degrade the system performance using resources unnecessarily.

There are a lot of challenges in proper triggering of cell switching in HetNets, particularly with high speed users. These challenges must be addressed carefully as they have direct bearing on the cell edge performance. It may be argued that HetNets are mostly expected in urban areas and high speed users do not exist in urban areas due to frequent red light stops. But in fact, in modern cities, highways and bullet trains can go through cities and a good number of users can move at high speed without making stops [2].

In a traditional macro cellular network, the radio link failures (RLFs) and ping-pong events can typically be avoided to a good extent [3]. However, the use of the set of cell switching procedures and parameters designed for a macro cellular network can degrade the mobility performance in HetNets [4], albeit the same set is used at present [5]. The poor mobility performance in HetNets with high speed users can be serious problems [6], [7], [8]. The authors of [4] show that the mobility performance strongly depends on the cell size and the user speed in HetNets. In [9], the authors show that the optimal value of cell switching parameters considering both the radio link failure rate and the ping-pong rate depends on the various cell sizes in HetNets. The authors of [10] show improvement in cell switching performance when the scaling of cell switching parameters considers both the user speed and the difference 
in cell sizes in HetNets. [10] also shows improvement in performance when the high speed users are primarily kept under only macro service, limiting the number of cell changes and the low speed users are offloaded to the small cells. The authors in [11] also proposed keeping the high speed users under the macro service but additionally consider the QoS of the application and so, three speed levels are used for the decision. The authors in [7] also proposed a context-aware mobility management procedure for HetNets, in which the resources are allocated to the users based on their velocities and historical data rates. The authors in [12] proposed network controlled macro cell mobility, while the small cell mobility is proposed to be UE autonomous. This offloads the network from having to perform frequent small cell change decisions, and reduces the signaling overhead. The authors in [13] proposed the adjustment of cell switching parameter values depending on whether the handover occurs from macro cell to small cell or from small cell to macro cell, since the conditions are different in these two cases. In [14], the authors attempt to determine appropriate cell switching parameter values considering handovers between macro and small cells and between macro and macro cells, separately. The authors in [15] proposed that the adjustment of cell switching parameters for a homogenous network is defined by a cost function that uses the rates of ping-pong, call drop and handover failure while assigning each of them weight factors. In [16] and [17], the authors also proposed similar cost functions but for HetNets and considering ping-pong, too late handover and too early handover. The authors of [18] show that if the adjustment of cell switching parameters with user velocity uses higher number of steps, then it improves the performance for a homogenous network.

It is thus already manifested that there are many different factors which should dictate the adjustment of cell switching parameters. Therefore, a versatile controllability should be used to accomplish such an adjustment. In this paper, we further investigate the challenges in mobility support for high speed users in HetNets and the investigation substantiates the necessity of versatile controllability. However, the existing specification or an existing proposal takes only one or two factors into account and therefore, the cell edge performance is at risk. We propose a scheme that attempts to overcome the current shortcomings.

The remainder of this paper is organized as follows. Interference issues in HetNets are investigated in Sec. 2. In Sec. 3, the existing cell switching conditions and the scaling of cell switching parameters for speedy users are presented. We delve into the challenges in mobility support for high speed users in HetNets in Sec. 4. In Sec. 5, we propose a scheme for adaptive scaling of cell switching parameters that uses enhanced and versatile controllability. Simulation results are given in Sec. 6 and the whole paper is concluded in Sec. 7.

\section{Interference Issues in HetNets}

We assume that the HetNets are using co-channel de- ployment, in which the small cells use the same spectrum as do the macro cells. Thus, the spectral efficiency is increased via spatial reuse but causing significant co-channel interference (CCI). Especially, the users in edge area of low power small cells are mostly vulnerable to strong interference signals from the high power macro cell. There are, in fact, two types of interference concerns: co-tier interference, which occurs between neighboring small cells, and cross-tier interference, which occurs between small cells and macro cells. The interference is a serious problem because of the following reasons.

1. A large number of cell boundaries are created in HetNets and there is a large difference between the transmit power levels of eNodeBs of macro cells and small cells, which makes the interference problem very complex. These interferences can arise in various scenarios as shown below [19].

i. The small cell users can be affected in downlink by the dominant macro eNodeBs.

ii. The small cell users can be affected in uplink by macro users. Also, the macro users can be affected in downlink by the small cell eNodeB. These interferences can be substantial when the macro users stay close to the small cells.

iii. The macro users can be affected in uplink by the small cell users. These interferences can be substantial when there are a large number of small cells deployed making the aggregate uplink interference from the large number of small cell users high.

iv. The small cell users can be affected both in downlink and in uplink by another small cell. These interferences can be substantial when the small cells are randomly located, which is more likely in residential deployments or in hot-spots. In enterprise deployments, small cells may be carefully located at predesigned places limiting the interference.

2. Since the eNodeB in the macro cell transmits much higher power than that in the small cell, the UE tends to remain connected to the macro cell even when the path loss between the UE and the small cell is smaller. This makes the load among tiers unbalanced. Therefore, 3GPP has standardized the provision for cell range expansion (CRE), which virtually increases the coverage area of small cells. In the case of CRE, by biasing handover decision toward a small cell, the users are handed over to the small cell earlier than usual and thus, some of the load is shifted from the macro cell to the small cell. Similarly, cell reselection can also be biased for users in RRC_IDLE state so that some additional users request services to the small cell when they need to. However, with CRE, the users, switched to small cells earlier than usual, receive low power from the serving small cell and high interference from the macro cell and thus, suffer from low SINR at the cell edge.

3. Many small cells, especially, femto cells are typically deployed in an ad hoc manner by users. They can 
even be moved or switched on or off at any time. Hence, traditional network planning and optimization becomes inefficient because operators do not control either the number or the location of these cells. The unplanned deployment aggravates the interference problem.

4. Some femto cells may operate in closed subscriber group (CSG) mode, in which cell access is restricted and only the respective subscribers are allowed to access the femto cells. The users, who are close to these femto cells but disallowed to access them, can be exposed to severe interference from them.

\section{Cell Switching Conditions}

In the current $3 \mathrm{GPP}$ specifications, the cell switching procedure is the same for a macro cellular network and for HetNets. The conditions for triggering cell reselection or handover are explained below and they are required to be fulfilled neither too early nor too late. The neighbor cells are considered to operate at intra-frequency or at interfrequency with equal priority.

\subsection{Conditions for Cell Reselection}

The UE evaluates the rank of the serving cell and the target neighbor cell frequently as

$$
R_{\mathrm{S}}=Q_{\text {meas'S }}+Q_{\text {hyst }} \text { and } R_{\mathrm{T}}=Q_{\text {meas'T }}-Q_{\text {offset }}
$$

where $Q_{\text {meas's }}$ and $Q_{\text {meas' }}$ are the signal strength of the serving cell and the target neighbor cell, respectively, measured as reference signal received power (RSRP) in $\mathrm{dBm} . Q_{\text {hyst }}$ is called the hysteresis margin. $Q_{\text {offset }}$ is a cell specific offset, which allows biasing the cell switching decision toward particular cells. If the UE finds that the target cell has been continuously better ranked for $T_{\text {reselectionRAT }}$ period, it reselects to the target cell. A separate $T_{\text {reselectionRAT }}$ timer is started for each cell that becomes better ranked than the serving cell. The UE reselects to the highest ranked cell when the corresponding timer expires. The eNodeB configures the values of $Q_{\text {hyst }}, Q_{\text {offset }}$ and $T_{\text {reselectionRAT }}$ using system information messages.

\subsection{Conditions for Handover}

The UE sends measurement reports frequently to the eNodeB, which convey the signal strength of the serving cell and the neighbor cells. Typically, the serving eNodeB issues a handover command after a certain number of measurement reports are received from the UE, indicating the target cell is better than the serving cell by a sufficient margin. The measurement reporting can be either eventtriggered or periodic as shown below.

- Event-Triggered: The UE is considered to enter and to leave a particular event when certain conditions are met. The UE begins sending measurement reports when the entering condition is met for TimeToTrigger period. As long as the event remains activated, the UE keeps on sending measurement reports at a certain interval until it reaches a maximum number of reports. There are five types of events indicated as A1 through A5. The whole entering condition for event A1 and event A3 are shown below for exemplification. The entering condition includes hysteresis for all five events.

Event A1:

Serving cell measurement - Hysteresis $>$ Threshold

Event A3:

Neighbor cell measurement

+ OffsetFreq for neighbor cell

+ CelllndividualOffset for neighbor cell

- Hysteresis > Serving cell measurement

+ OffsetFreq for serving cell

+ CelllndividualOffset for serving cell

+ A3_Offset

- Periodical: The UE keeps on sending the measurement reports one after another at a certain interval until it reaches a maximum number of reports.

As the UE moves, if the handover occurs too late, the received power from the source cell can fall too low. This can cause RLF. The RLF can lead to a handover failure (HF). When the HF occurs due to RLF, usually, the UE fails to receive RRCConnectionReconfiguration message from the source cell, which carries instruction to switch to the target cell. After the HF, the UE can attempt to reestablish RRC connection with the strongest cell. A call or session drop occurs if this reestablishment fails. If the RRC connection reestablishment becomes successful, the target cell can send a RLF indication message to the source cell.

As the UE moves, if the handover occurs too early, the radio link with the target cell may not have become sufficiently strong yet. In this case, during or after the handover procedure, RLF can occur at the target cell and then the UE tries to reestablish RRC connection with the old source cell. After the RRC connection reestablishment, the source cell can send a RLF indication message to the target cell. To inform the source cell about the reason for failure, the target cell can send a handover report message indicating 'handover too early'.

\subsection{Scaling of Parameters for Speedy Users}

The high speed users may be subject to very poor SINR due to what is often called a dragging effect. This is because the cell switching is not triggered until the required conditions have remained fulfilled for a certain period. This delay would let the speedy users move too far away from the old serving cell before switching to the target cell. This results in poor signal strength from the serving cell and strong interference from the target cell. Thus, the dragging effect causes poor cell edge SINR and extremely low data rate and in the worst case, it can cause RLF and HF. As a remedy to this problem, 3GPP standardizes a scaling of cell switching parameters for speedy users. Accordingly, 
apart from the normal-mobility state, the medium and high mobility states are defined as shown below and scaling is applied only in these two states.

- High-Mobility State: The UE enters this state if there are more than $N_{\mathrm{CR}_{-} \mathrm{H}}$ numbers of cell reselections or handovers in the last $T_{\mathrm{CRmax}}$ duration indicating high user speed.

- Medium-Mobility State: The UE enters this state if there are more than $N_{\mathrm{CR} \text { M }}$ number but equal to or less than $N_{\mathrm{CR} \text { H }}$ number of cell reselections or handovers in the last $T_{\mathrm{CRmax}}$ duration indicating medium user speed.

- Normal-Mobility State: The UE enters this state from medium or high mobility state if neither medium nor high mobility state is detected during $T_{\text {CRmaxHyst }}$ period. No scaling is performed in this state.

The eNodeB configures the values of $T_{\mathrm{CRmax}}, N_{\mathrm{CR} H}$, $N_{\mathrm{CR} \text { M }}$ and $T_{\text {CRmaxHyst }}$. In the case of cell reselection, the UE scales $Q_{\text {hyst }}$ and $T_{\text {reselectionRAT }}$ as follows.

- $Q_{\text {hyst }}$ : In high and medium mobility states, SF-High and SF-Medium fields are added to $Q_{\text {hyst }}$, respectively. The eNodeB sends these two fields on Q-HystSF IE via system information message and their values can be $-6,-4,-2$ or $0 \mathrm{~dB}$.

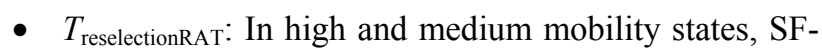
High and SF-Medium fields are multiplied with $T_{\text {reselectionRAT }}$, respectively. The eNodeB sends these two fields on SpeedStateScaleFactors (SSSF) IE via system information message and their values can be $0.25,0.5,0.75$ or 1 .

In the case of handover, the hysteresis is not scaled in the current specifications. In high and medium mobility states, SF-High and SF-Medium fields are multiplied with TimeToTrigger, respectively. The eNodeB sends these two fields on SSSF IE via RRCConnectionReconfiguration message.

\section{Challenges in Mobility Support in HetNets}

The challenges in mobility support for speedy users in HetNets are manifold and we derive them as follows.

1. Section 2 explains that SINR can be very poor at the cell edge of small cells even for immobile users in HetNets. On top of that the dragging effect is particularly severe in a HetNet scenario with high speed users [2], which further aggravates the cell edge SINR. Thus, the cell edge performance degrades severely and there can be even RLF and HF.

2. Typically, the lowest received signal power is experienced in the system right before the cell switching and this power is denoted as $P_{\mathrm{R}_{-} \text {Min. }}$. Currently, a scaling cell switching parameters is performed for speedy users but only at two discrete steps as shown in Sec. 3.3.

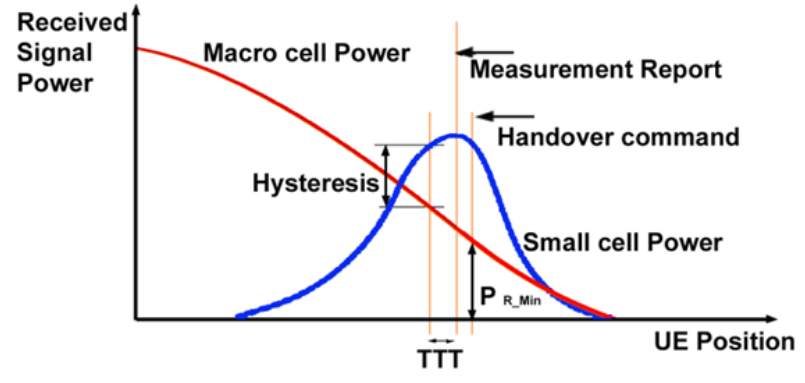

Fig. 1. Reception of low $P_{\mathrm{R}_{\_} \text {Min }}$ for speedy users in HetNets.

Thus, for speedy users in HetNets, the cell switching may not be triggered as quickly as it is required and the UE can move far inside the new cell before the new cell takes over as illustrated in Fig. 1. Here, $P_{\mathrm{R}_{-} \text {Min }}$ is not enough velocity independent and $P_{\mathrm{R}_{\_} \text {Min }}$ can fall very low. This can cause highly degraded data rate in the case of handover and there can be even RLF and HF. Similar problem can also occur in the case of cell reselection.

3. The receiver sensitivities for the eNodeB and the UE are required to support $P_{\mathrm{R}_{-} \text {Min }}$ in uplink and downlink, respectively. But $P_{\mathrm{R} \text { Min }}$ takes on widely varying values at different velocities with only two steps of scaling. This may pose difficulty to match between the receiver sensitivity and $P_{\mathrm{R} \_ \text {Min }}$ in the system.

4. In HetNets, the existence of high number of cells can lead to a lot of overlapping in cell coverage. Also, there can be multiple cell borders and different target cells at different times, due to the fast variation of SINR from each cell [2]. Thus, the cell boundaries and target cells can be far from clear and there can be difficulties in successful cell switching.

5. The existing method relies merely on the history of cell switching for the decision of scaling. But this history is not always a good indication of the user speed. For example, a user at a low velocity may take a tangled pathway within overlapped cells and undergo frequent cell switching. Then the existing method applies high scaling, which is not justified because a sufficient margin is required to avoid pingpong effects. In this case, if the high scaling was not applied, the UE would not go far inside another cell. Thus, the possibility of ping-pong effects is unnecessarily increased.

6. In HetNets, the adjustment of cell switching parameters should consider the different characteristics of the neighbor cells. For switching from small cells to macro cells, the received power from the small cell drops much quicker compared to cell switching between macro cells. Thus, a late cell switching can lead to very poor SINR. For cell switching from macro cells to small cells, the received power from the small cell increases much quicker compared to cell switching between macro cells and the UE suffers from inter-cell interference from the small cell earlier. Thus, a late cell switching can again lead to very poor SINR. 
7. The handover conditions should be adaptive to fit into the current loading conditions and this adaptability is not enough effective at present. The CRE can vary its extent depending on loading conditions. Moreover, the SINR on PDCCH depends on the traffic usage pattern. Since HARQ cannot be applied to PDCCH, if the BLER on PDCCH exceeds $10 \%$ during handover, the UE can fail to receive response from the eNodeB. This causes HF. The impact of traffic pattern on SINR on PDCCH is explained below.

A small number of high data rate users, using applications like FTP, get PDCCH lightly loaded. On the other hand, PDCCH gets heavily loaded if there are a large number of low rate users in the cell, using applications like VoIP. A PDCCH instance uses a number of control channel elements (CCEs) where a CCE consists of nine resource element groups (REGs) or 36 resource elements. There are four different $\mathrm{PDCCH}$ formats that use different aggregation levels of CCEs. A higher aggregation level with a larger number of CCEs can be used to achieve stronger coding for cell edge users with poor SINR condition. But a high aggregation level may be difficult to be made available when the PDCCH loading is high. Besides, a light loading on PDCCH allows less co-channel interference from neighboring cells. This is because the whole control region is not used with the light loading and the symbol quadruplets of the CCEs from each cell are shifted to different sub-carrier frequencies or different symbols to randomize and reduce co-channel interference [1], [2].

8. In HetNets, the downlink coverage of a small cell is much smaller than that of a macro cell. But this is not the case for uplink; since all UEs have almost equal transmit power capabilities. This can create a mismatch between downlink and uplink handover boundaries compared to homogenous networks, in which those boundaries are more closely matched [20]. Since the handover decision is based on the downlink boundaries, a UE with ongoing data transfer only in uplink may not trigger handover at a very appropriate moment in HetNets. For example, a UE moving from small cell to macro cell and undergoing uplink data transfer will have to boost the uplink transmit power suddenly once the handover is triggered because the macro eNodeB is far away. This will cause additional interference, which could be avoided if the handover was triggered later. Similarly, if the UE was moving from macro cell to small cell, an earlier handover could preclude high uplink power transmission recently before the handover and its associated interference.

\section{Proposed Scheme}

It can be inferred from the discussion in Sec. 4 that a versatile controllability over cell switching is required to address the wide variety of challenges. We propose the following changes in the scheme of scaling of cell switching parameters.

i. A versatile controllability over the adaptive scaling is used so that the scaling can better fit into the current status considering user speeds, traffic loads, street patterns and types of cells involved in switching.

ii. The user velocity $v$ and the maximum Doppler shift $f_{\mathrm{d}}$ are related as $f_{\mathrm{d}} \lambda$ where $\lambda$ is the wavelength. In order to improve versatility in the controllability, instead of using only the recent history of cell switching for computation of the scaling factor, it is combined with the Doppler spread, which is also indicative of the user speed. The rationale behind this action is that one between the number of cell switching and the estimated Doppler spread can be found better indicative of the requirement of scaling than the other depending on the scenario in HetNets. Secondly, since the scaling with speed reduces the cell switching delay, it increases the possibility of ping-pong effects. The inclusion of Doppler spread ensures that the velocity is really high when high scaling is applied and the chances of ping-pong effects are thus mitigated.

iii. The scaling varies smoothly with the user speed instead of varying only at two discrete steps. The medium mobility state is removed and $N_{\text {CR_M }}$ is not transmitted. A smooth scaling is performed in the high mobility state and the normal mobility uses no scaling. Because of the smooth scaling, $P_{\mathrm{R} \text { Min }}$ becomes more independent of the user speed. Thus, $P_{\mathrm{R} \_ \text {Min }}$ does not fall too low providing better SINR and this may prevent RLF and HF. Also, due to the stability of $P_{\mathrm{R} \_ \text {Min }}$, the receiver can be more easily designed with proper sensitivity.

iv. Instead of letting eNodeB gain the whole control over scaling, a part of the control is shifted to the UE. The eNodeB controlled scaling of the hysteresis margin is used to establish a set point. Then the UE performs adjustment of timer period as an overlay around the set point at the discretion of the UE itself. The adjustment by the UE does not require any feedback or any overhead and it can quickly adapt to the changes. Thus, it can function as a fine-tuning on top of the eNodeB controlled scaling.

Several methods have been suggested for the estimation of Doppler spread for OFDM signals [21], [22], [23] and the UE may use any reliable method in the proposed scheme. [21] uses the relationship

$$
v=f_{\mathrm{d}} \lambda=\frac{c f_{\mathrm{d}}}{f_{\mathrm{c}}}=\frac{2.405 c}{2 \pi f_{\mathrm{c}} T_{S} \hat{l}_{0}}
$$

where $f_{\mathrm{c}}$ is the signal frequency, $c$ is the speed of light, $T_{\mathrm{S}}$ is OFDM symbol duration and $\hat{\vartheta}_{0}$ is the zero crossing point of the estimated covariance function, $\varphi_{\mathrm{t}}(l)$ of the received signal at a certain carrier in the frequency domain with $l$ representing the difference in time. The velocity or Doppler spread estimation is shown almost independent on SINR in 


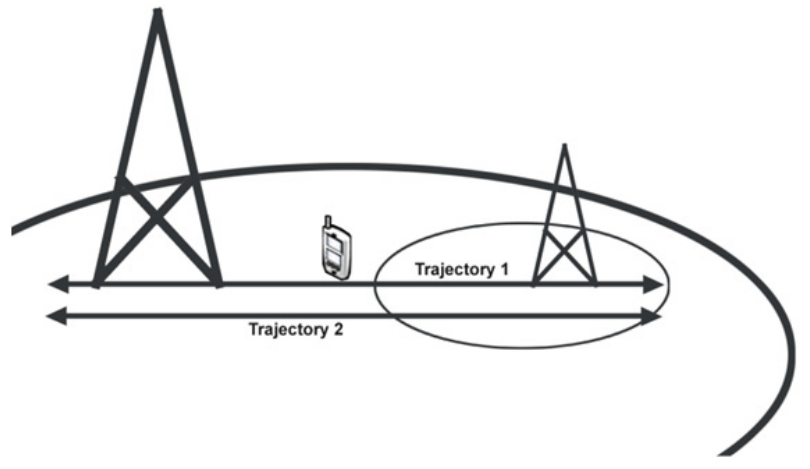

Fig. 2. The trajectory along which the user moves.

[21] and so, it can be used at the cell edge during cell switching.

We assume that the user moves along trajectory 1 in Fig. 2 in any direction at velocity $v$ where trajectory 1 is a straight line connecting the eNodeBs of macro cell and small cell. $D_{\mathrm{S}}$ and $D_{\mathrm{T}}$ are the distances of the cell border from the serving eNodeB and the target eNodeB, respectively. The proposed scaling scheme is explained in Sec. 5.1 and 5.2 for cell reselection and handover, respectively.

\subsection{Scheme for Cell Reselection}

The proposed scaling of hysteresis and timer period for cell reselection is depicted in Fig. 3. We assume that the condition for the triggering of $T_{\text {reselectionRAT }}$ timer is met at a distance $d$ from the cell border of the target cell. According to the log distance path loss model, the received power at $d$ from the serving cell and the target cell can be expressed in $\mathrm{dB}$ as

$\overline{P r_{\mathrm{S}}}\left(d_{0}\right)-10 n \log \left(\frac{D_{\mathrm{S}}+d}{d_{0}}\right)$ and $\overline{P r_{\mathrm{T}}}\left(d_{0}\right)-10 n \log \left(\frac{D_{\mathrm{T}}-d}{d_{0}}\right)$

respectively, where $n$ is the path loss exponent for the multipath environment. Ignoring the quantization in power level calculation, (1) can be shown as

$$
\begin{array}{r}
10 n \log \left(\frac{D_{\mathrm{S}}+d}{d_{0}}\right)-10 n \log \left(\frac{D_{\mathrm{T}}-d}{d_{0}}\right) \\
=Q_{\text {hyst }}+\overline{P r_{\mathrm{S}}}\left(d_{0}\right)-\overline{\operatorname{Pr}_{r_{\mathrm{T}}}}\left(d_{0}\right)
\end{array}
$$

where $Q_{\text {offset }}$ has been set to zero assuming zero bias to the target cell. Equation (3) can be rearranged as

$$
4.343 n\left[\ln \left(1+\frac{d}{D_{\mathrm{S}}}\right)-\ln \left(1-\frac{d}{D_{\mathrm{T}}}\right)+\ln \left(\frac{D_{\mathrm{S}}}{D_{\mathrm{T}}}\right)\right]-\Delta_{\mathrm{Pr}}\left(d_{0}\right)=Q_{\text {hyst }}
$$

where $\Delta_{\mathrm{Pr}}\left(d_{0}\right)=\overline{P_{r_{\mathrm{S}}}}\left(d_{0}\right)-\overline{P_{r_{\mathrm{T}}}}\left(d_{0}\right) \cdot \Delta_{\mathrm{Pr}}\left(d_{0}\right)$ will be positive when the UE is moving from macro cell to small cell and it will be negative when the UE is moving from small cell to macro cell. Using Taylor series expansion, assuming $d<<D_{\mathrm{S}}$ and $d<<D_{\mathrm{T}}$ and ignoring higher order terms

$$
4.343 n\left(\frac{1}{D_{\mathrm{S}}}+\frac{1}{D_{\mathrm{T}}}\right) d+4.343 n \ln \left(\frac{D_{\mathrm{S}}}{D_{\mathrm{T}}}\right)-\Delta_{\mathrm{Pr}}\left(d_{0}\right)=Q_{\text {hyst }}
$$

$4.343 n \ln \left(D_{\mathrm{S}} / D_{\mathrm{T}}\right)-\Delta_{\mathrm{Pr}}\left(d_{0}\right)$ is a constant for a particular scenario. To attain smooth scaling, we proposed to be reduced linearly as the user velocity $v$ increases and for this purpose, $Q_{\text {hyst }}$ can be scaled as

$$
4.343 n\left(\frac{1}{D_{\mathrm{S}}}+\frac{1}{D_{\mathrm{T}}}\right) d+4.343 n \ln \left(\frac{D_{\mathrm{S}}}{D_{\mathrm{T}}}\right)-\Delta_{\mathrm{Pr}}\left(d_{0}\right)=Q_{\mathrm{hyst}}-k^{\prime} v
$$

$k$ ' in (6) would be configured by the eNodeB using the parameter $Q_{\text {hyst }} S F$ where $Q_{\text {hyst }} S F$ is negative in $\mathrm{dB}$ value as given by

$$
\begin{aligned}
& 4.343 n\left(\frac{1}{D_{\mathrm{s}}}+\frac{1}{D_{\mathrm{T}}}\right) d+4.343 n \ln \left(\frac{D_{\mathrm{s}}}{D_{\mathrm{T}}}\right)-\Delta_{\mathrm{Pr}}\left(d_{0}\right) \\
& =Q_{\mathrm{hyst}}+Q_{\mathrm{hyst}} S F \cdot k v .
\end{aligned}
$$

The UE determines a factor Vel_factor_CR, proportional to $v$, and uses it in place of $k v$ as

$$
\begin{gathered}
4.343 n\left(\frac{1}{D_{\mathrm{S}}}+\frac{1}{D_{\mathrm{T}}}\right) d+4.343 n \ln \left(\frac{D_{\mathrm{S}}}{D_{\mathrm{T}}}\right)-\Delta_{\mathrm{Pr}}\left(d_{0}\right) \\
=Q_{\text {hyst }}+Q_{\text {hyst }} S F \cdot V e l_{\text {_factor_C }} C R .
\end{gathered}
$$

Despite the deployment of HetNets, it may happen that the UE finds itself moving between two macro cells. Assuming all macro cells to have radius $D$ and equal transmit power at eNodeBs, a similar derivation yields

$$
\frac{8.686 n}{D} d=Q_{\text {hyst }}+Q_{\text {hyst }} S F \cdot V e l_{-} \text {factor_CR. }
$$

The Vel_factor_CR is computed from the number of cell reselections in the last $T_{\mathrm{CRmax}}$ period, $N_{\text {cr }}$ and the estimated Doppler spread as

$$
\text { Vel_factor_CR }=(1-\alpha) N_{\text {cr filtered }_{\text {f }}}+\alpha \beta f_{\mathrm{d}}
$$

where

$$
\begin{gathered}
N_{\text {cr filtered }_{\text {f }}}=(1-\gamma) N_{\text {cr }}+\gamma N_{\text {cr_last }} \\
\gamma=1 / 2^{\eta} .
\end{gathered}
$$

Here, $N_{\text {cr filtered }}$ represents the filtered value of $N_{\text {cr }}$ and it is updated in every $T_{\mathrm{CRmax}}$ period. $N_{\text {cr last }}$ gives the last filtered value of $N_{\mathrm{cr}} . \eta$ is the filter coefficient, which can vary between 0 and 6 . It is expected that the impact of $N_{\mathrm{cr}}$ is more important than $N_{\text {cr last }}$ and $\eta$ will generally be high. (11) and (12) allow better granularities for the impact of $N_{\mathrm{cr}}$ for higher values of $\eta . B$ is a gain factor and the value of $\beta$ should be chosen to yield the same order of values for $N_{\text {cr filtered }}$ and $\beta f_{\mathrm{d}} . \beta$ decreases with $f_{\mathrm{c}}$ and so, it will vary with the operating band. $\alpha$ is used to control the relative influence of the number of cell reselections and Doppler spread. The UE applies $Q_{\text {hyst_scaled }}$ as the scaled hysteresis, which is computed as

$$
Q_{\text {hyst_scaled }}=Q_{\text {hyst }}+\left(Q_{\text {hyst }} S F \cdot V e l_{-} \text {factor_CR }\right)_{-} \text {. }
$$




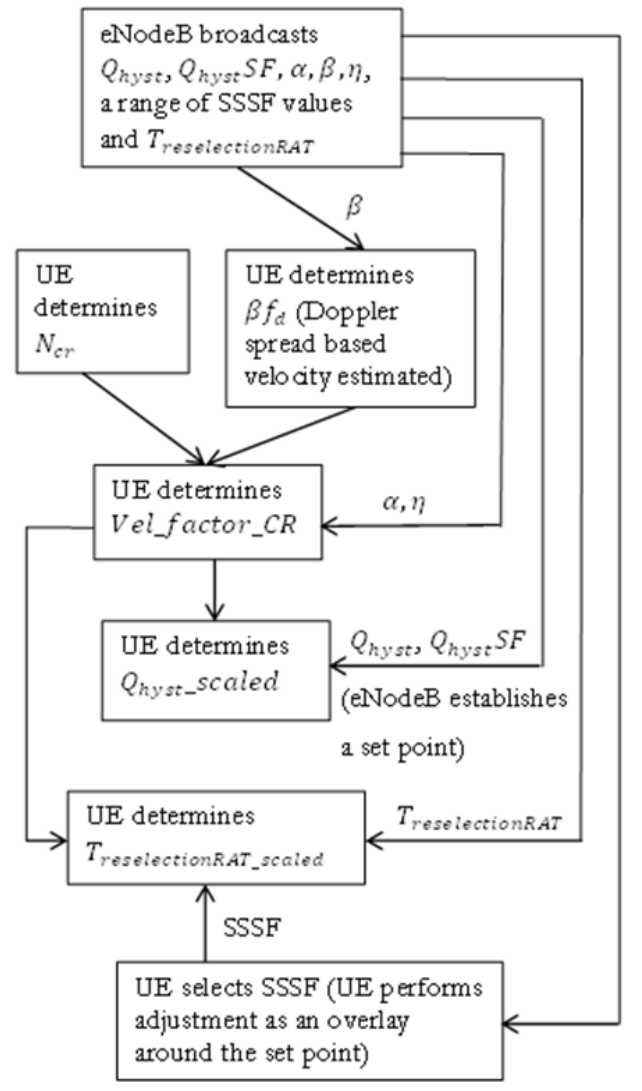

Fig. 3. A flowchart showing determination of scaled parameters for cell reselection.

Once the condition of (13) is fulfilled at a distance $d$, the timer $T_{\text {reselectionRAT }}$ is supposed to trigger and run over an additional distance $d$ ' before triggering cell reselection where

$$
d^{\prime}=v \cdot T_{\text {reselectionRAT }} .
$$

To keep $d$ ' reasonably constant, the UE uses a scaled timer period in place of $T_{\text {reselectionRAT, which is given by }}$

$$
T_{\text {reselectionRAT_scaled }}=T_{\text {reselectionRAT }} \frac{S S S F}{V e l_{-} \text {factor_CR }} .
$$

The eNodeB transmits $Q_{\text {hyst }} S F, \alpha, \beta, \eta$ and a range of values for SSSF using system information message. The UE selects $S S S F$ value from the given range.

\subsection{Scheme for Handover}

We propose a method of scaling for handover similar to what is explained for cell reselection in Sec. 5.1. We assume event-triggered measurement reporting. Vel factor_HO, a factor proportional to the user velocity is computed from the number of handovers in last $T_{\mathrm{CRmax}}$ period, $N_{\text {ho }}$ and the estimated Doppler spread as

$$
\begin{aligned}
& \text { Vel_factor_ } H O=(1-\alpha) N_{\text {ho filtered }}+\alpha \beta f_{\text {d }} \\
& \text { where } \quad N_{\text {ho_filtered }}=(1-\gamma) N_{\text {ho }}+\gamma N_{\text {ho_last }} \\
& \gamma=1 / 2^{\eta}
\end{aligned}
$$

The hysteresis in the entering conditions for eventtriggered measurement reporting is not scaled with the user speed in the existing method. But we propose that the scaling of hysteresis is performed and thus, a set point for scaling is established. The UE applies Hysteresis_scaled as the scaled hysteresis, which is computed as

$$
\begin{aligned}
& \text { Hysteresis_scaled }= \\
& \text { Hysteresis }+(\text { Hyst_SF.Vel_factor_HO })
\end{aligned}
$$

where Hyst_SF is negative in $\mathrm{dB}$ value. The distance $d$ " over which the entering conditions for a particular event need to be met can be expressed as

$$
d^{\prime \prime}=v \cdot \text { TimeToTrigger } .
$$

To keep $d$ " reasonably constant, the UE uses scaled timer period given by

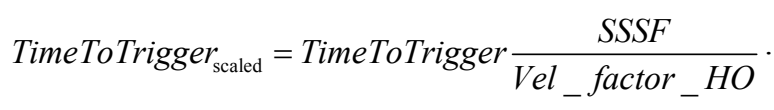

The eNodeB transmits Hyst_SF, $\alpha, \beta$, $\eta$, and a range of values for SSSF using RRCConnectionReconfiguration message. The UE selects $S S S F$ value from the given range.

\subsection{Control over Scaling}

The proposed scheme provides versatile controllability over the scaling depending on the scenario. The scenarios in HetNets may vary widely as a result of differences in transmit power, coverage of macro and small cells, traffic loading in macro and small cells, position and traffic pattern of highways and other streets with respect to the coverage of macro and small cells, profile of the user speeds, operating frequency, multipath environment and fading conditions, QoS requirements of data services, and so forth. A dynamic control is proposed that uses $Q_{\text {hyst }} S F$ or Hyst_SF, SSSF, $\alpha$ and $\eta$ based on various factors of the particular scenario and for this purpose, some guidelines are given below. The operator may not necessarily limit to these guidelines but may further extend, if required.

1. The scaling of hysteresis establishes a set point using $Q_{\text {hyst }} S F$ or Hyst_SF, configured by the eNodeB. The scaling of timer period provides an overlay of scaling around the set point using $S S S F$, configured by the UE. Increasing the absolute values of $Q_{\text {hyst }} S F$ or Hyst $S F$ reduces hysteresis and thus, yields a quicker cell switching. On the other hand, increasing the value of SSSF increases timer period and thus, yields a delayed cell switching. Simultaneous control from both the eNodeB and the UE may often reduce the controllability. Therefore, $S S S F$ must be within a range of values, provided by the eNodeB.

In the case of handover, Hyst_SF and SSSF values are adjusted based on too late handover, too early handover, and ping-pong events. Section 3.2 shows how too early handover and too late handover can be detected from different messages. Ping-pong events can be detected from the identity of the recently serving 
cells. Similar with the proposal in [17], the adjustment of Hyst_SF uses a cost function as

$$
A d j_{\text {Hyst_SF }}=w_{1} \frac{N_{1}}{N_{\mathrm{T}}}-w_{\mathrm{e}} \frac{N_{\mathrm{e}}}{N_{\mathrm{T}}}-w_{\mathrm{p}} \frac{N_{\mathrm{P}}}{N_{\mathrm{T}}}
$$

where $N_{\mathrm{l}}, N_{\mathrm{e}}, N_{\mathrm{p}}$, and $N_{\mathrm{T}}$ are the numbers of too late handovers, too early handovers, ping-pong events, and total handovers in last $T_{\mathrm{ho}}$ period and $w_{\mathrm{l}}, w_{\mathrm{e}}$, and $w_{\mathrm{p}}$, are their respective weight factors. $T_{\mathrm{ho}}$ is configured by the eNodeB. Hyst_SF is updated as

$$
\left|H y s t_{-} S F_{\text {new }}\right|=\left|H y s t_{-} S F+A d j_{\text {Hyst_SF }}\right| .
$$

The above adaption of scaling of hysteresis will be slow in comparison with the fast changes for speedy users in HetNets. But the overlay adjustment by the UE is quick and so, it can take care of instant requirements. The UE will slightly decrease SSSF whenever it detects a too late handover and the UE will slightly increase SSSF whenever it detects a too early handover or a ping-pong event. In the case of cell reselection, the UE adjusts $Q_{\text {hyst }} S F$ and SSSF the way Hyst_SF and SSSF are adjusted in the case of handover. In the case of either handover or cell reselection, the UE detects a too late cell switching from RLF at the source cell and a too early cell switching from RLF at the target cell. The UE detects ping-pong events from the identity of the recently serving cells.

2. The eNodeBs are updated with the information of different cell sizes in HetNets. Then in RRC CONNECTED state, the serving eNodeB can determine from the measurement report whether the UE is moving from macro cell to small cell, from small cell to macro cell or between two macro cells. A higher absolute value of Hyst_SF can be used if the UE is moving between a macro cell and a small cell compared to the movement between two macro cells. To adjust Hyst_SF for this purpose, the eNodeB can send a quick update to the UE.

3. When the UE performs data transfer only in the uplink, to limit increase in the uplink power transmission, the eNodeB can send a quick update to set a lower absolute value of Hyst_SF if the UE is moving from small cell to macro cell. Similarly, an update to set a higher value of Hyst_SF can be used if the UE is moving from macro cell to small cell. However, this can lead to poor received power in downlink but the strong modulation and coding scheme (MCS) used for PCFICH, PDCCH and PHICH may still enable their successful decoding.

4. $\alpha$ is used to control the relative influence of the number of cell switching and Doppler spread in the computation of the scaling factor. If the traffic pattern of the streets suggests that a user may keep varying his speed widely, a high $\alpha$ may be used. This is because the Doppler spread now better indicates the current state of the user. A low $\alpha$ may be used if there are frequent red light stops. In this case, although the users have low velocity, they have chances of ping-pong effects because they may stop or turn around [24]. Low $\alpha$ may also be used when the Doppler spread estimation in the physical layer is not very reliable.

5. When the UE finds that its own velocity, estimated from the Doppler spread, exceeds a threshold value $v_{\text {th }}$, it sets SSSF to a very high value $S S S F_{\text {high. }}$. This will preclude switching to small cells frequently and the cell switching will rather occur only between macro cells. $v_{\text {th }}$ and $S S S F_{\text {high }}$ are configured by the eNodeB.

6. When there is high PDCCH loading, a tighter control over handover timing can be used. And for this purpose, the required number of measurement reports with the information of a better neighbor cell, to issue a handover command, can be adjusted using a cost function similar with (22).

7. The filter coefficient $\eta$ is used to control the relative influence between the recent and older number of cell switching and its value should be set accordingly. The filtering removes sudden errors in the estimation. An example of such error can be that a highway may take a turn around the eNodeB and the user at a high velocity may not undergo cell changes for a while.

\section{Simulation}

A MATLAB based simulation has been performed and the simulation environment is limited to partially implement the proposed features. Primarily, the simulation manifests the performance due to the smoothness in scaling in the proposed scheme and compares it with the two discrete steps of scaling in the existing scheme. A strong system level simulator is required for comprehensive validation of the proposals. But such a simulator cannot be made accessible at the moment and so, a proper simulation has been left as a future work. However, Section 5 logically explains that the proposed scheme has ample and versatile controllability and it can potentially have remarkable achievements. The simulation assumptions are shown in Tab. 1 . The parameter values in Tab. 1 comply with 3GPP specifications.

In the limited simulation environment, the simulation results are not significantly different between the movements from macro cell to small cell and from small cell to macro cell. Therefore, the simulation results are shown only for the user moving from macro cell to small cell along the two straight line trajectories of Fig. 2. Trajectory 2 is a straight line parallel with Trajectory 1 at $150 \mathrm{~m}$ distance. The user moves along Trajectory 2 only in the simulation results of Fig. 6 while Trajectory 1 is used in all other figures in this section. The simulation was performed for a number of fixed user velocities, against which $P_{\mathrm{R}_{-} \text {Min }}$ was plotted. $P_{\mathrm{R}_{-} \text {Min }}$ was calculated as the received power from the source cell at the position where cell switching is triggered. The path loss was calculated using OkumuraHata model. $N_{\text {cr }}$ and $N_{\text {ho }}$ were calculated as the average 


\begin{tabular}{|c|c|}
\hline Parameter & Value \\
\hline Operating frequency (DL) & $1700 \mathrm{MHz}$ \\
\hline $\begin{array}{l}\text { Separation between trajectory } 1 \\
\text { and } 2\end{array}$ & $150 \mathrm{~m}$ \\
\hline User velocity (constant) & $10,20,30, \ldots, 150 \mathrm{~km} / \mathrm{hr}$ \\
\hline User direction & $\begin{array}{l}\text { Moving from macro cell to small } \\
\text { cell }\end{array}$ \\
\hline Path loss model & Okumura-Hata \\
\hline EIRP at macro eNB & $40 \mathrm{dBm}$ \\
\hline EIRP at smalleNB & $23 \mathrm{dBm}$ \\
\hline $\begin{array}{l}\text { Separation between macro eNB } \\
\text { and small eNB }\end{array}$ & $800 \mathrm{~m}$ \\
\hline Antenna height at macro eNB & $30 \mathrm{~m}$ \\
\hline Antenna height at small eNB & $20 \mathrm{~m}$ \\
\hline User antenna height & $1 \mathrm{~m}$ \\
\hline$T_{\mathrm{CR \operatorname {max }}}$ & $4 \mathrm{sec}$ \\
\hline$T_{\text {reselectionRAT }}$ & $6 \mathrm{sec}$ \\
\hline$Q_{\text {offset }}$ & $0 \mathrm{~dB}$ \\
\hline$Q_{\text {hyst }}$ & $10 \mathrm{~dB}$ \\
\hline SF-High $(Q-H y s t S F)$ & $4 \mathrm{~dB}$ \\
\hline SF-Medium $(Q-H y s t S F)$ & $8 \mathrm{~dB}$ \\
\hline SF-High $(S S S F)$ & 0.75 \\
\hline SF-Medium $(S S S F)$ & 0.25 \\
\hline$Q_{\text {hyst }} S F$ (proposed case) & $-0.2,-0.4,-0.6$ or $-0.8 \mathrm{~dB}$ \\
\hline Reporting method for $\mathrm{HO}$ & Event-triggered with event A3 \\
\hline $\begin{array}{l}\text { CellIndividualOffset } \\
\text { for neighbor cell }\end{array}$ & $0 \mathrm{~dB}$ \\
\hline $\begin{array}{l}\text { CellIndividualOffset } \\
\text { for serving cell }\end{array}$ & $0 \mathrm{~dB}$ \\
\hline A3_Offset & $2 \mathrm{~dB}$ (IE value, 4 ) \\
\hline Hysteresis & $8 \mathrm{~dB}$ (IE value, 16) \\
\hline ReportInterval & $0.24 \mathrm{sec}$ \\
\hline No. of reports triggering $\mathrm{HO}$ & 4 \\
\hline Hyst_SF(proposed case) & -0.4 or $-0.7 \mathrm{~dB}$ \\
\hline TimeToTrigger & $2.56 \mathrm{sec}$ \\
\hline$N_{\mathrm{CR} \_\mathrm{M}}$ (existing case) & 3 \\
\hline$N_{\mathrm{CR} \_\mathrm{H}}$ (existing case) & 7 \\
\hline$N_{\mathrm{CR} \text { H }}$ (proposed case) & 2 \\
\hline$S S S F$ (proposed case) & $0.5,1,1.5$ or 2 \\
\hline$\alpha$ & 0.4 \\
\hline$\beta$ & 4 \\
\hline$\eta$ & 3 \\
\hline
\end{tabular}

Tab. 1. Simulation assumptions.

number of cell reselections or handovers in the last $T_{\mathrm{CR} \max }$ period for a particular user velocity. For simplicity, $N_{\text {cr last }}$ or $N_{\text {ho last }}$ were also calculated the same way but using immediate lower velocity from the velocity array. $f_{\mathrm{d}}$ was calculated as $f_{\mathrm{d}}=v / \lambda$ and $\lambda$ was calculated as $c / f$ based on the operating downlink frequency. Fixed values of $\alpha, \beta$ and $\eta$ were used in all simulations because the simplified simulation environment is unable to demonstrate discrepancies with variation in their values. The simulation results are shown in Sec. 6.1 and 6.2 for cell reselection and handover, respectively.

\subsection{Results for Cell Reselection}

Figures 4 to 6 show $P_{\mathrm{R} \text { Min }}$ vs. user speed profile for different values of SSSF comparing existing and proposed methods in the case of cell reselection. In Fig. $4, P_{\mathrm{R} \text { Min }}$ follows a smooth variation and it remains fairly velocity independent in the proposed scheme. Conversely, $P_{\mathrm{R}_{-} \text {Min }}$

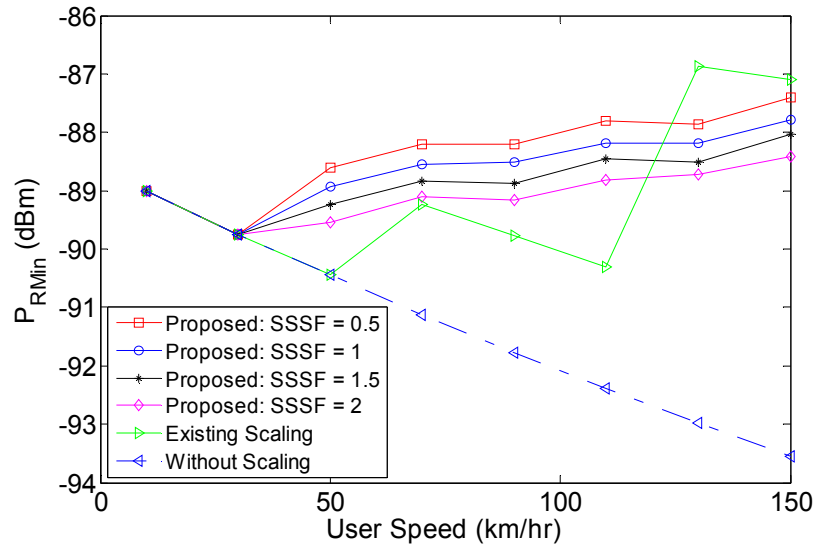

Fig. 4. $P_{\mathrm{R}_{-} \text {Min }}$ vs. user speed for $\mathrm{CR}\left(Q_{\text {hyst }} S F=-0.4 \mathrm{~dB}\right)$.

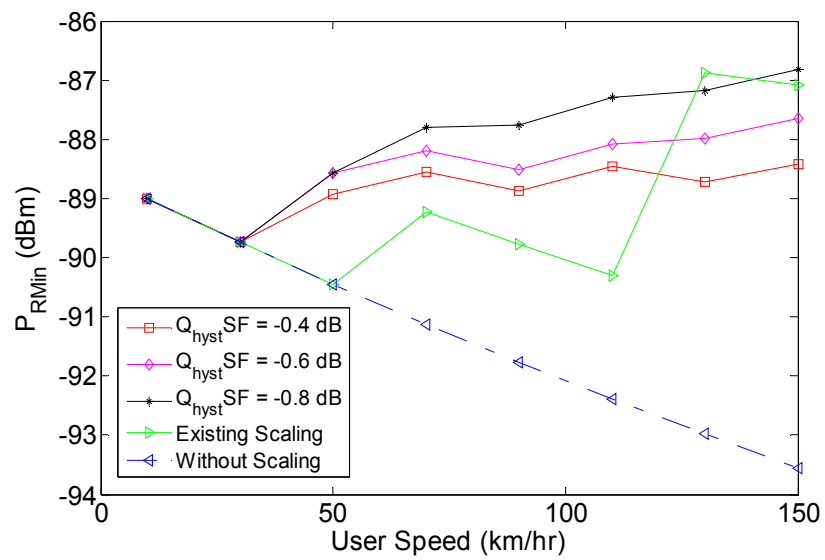

Fig. 5. $P_{\mathrm{R} \text { Min }}$ vs. user speed for $\mathrm{CR}$ with adaptation of SSSF by the UE for $Q_{\text {hyst }} S F=-0.4 \mathrm{~dB},-0.6 \mathrm{~dB}$ and $-0.8 \mathrm{~dB}$.

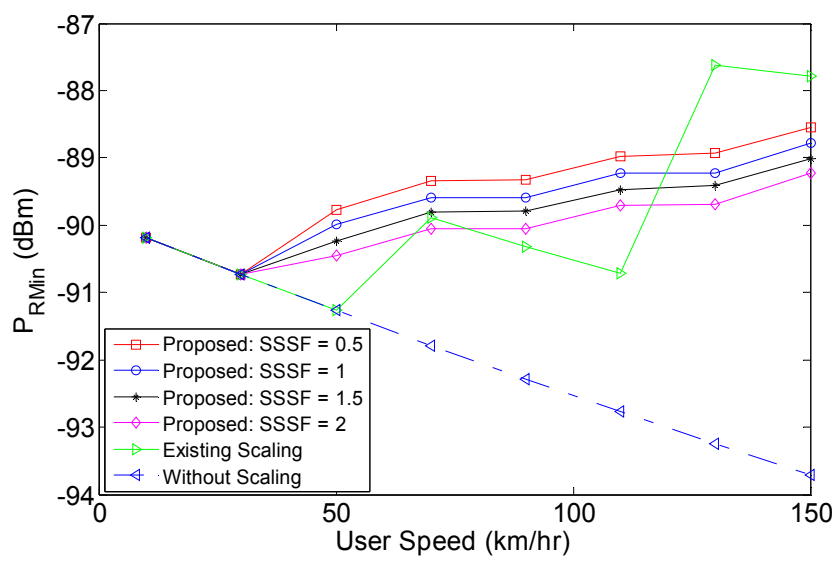

Fig. 6. $P_{\mathrm{R}_{-} \text {Min }}$ vs. user speed for $\mathrm{CR}\left(Q_{\mathrm{hyst}} S F=-0.4 \mathrm{~dB}\right.$, trajectory 2).

shoots within a wide range in the existing scheme. Here, $P_{\mathrm{R}_{-} \text {Min }}$ shoots when there is a change in mobility state. Ordinarily, $P_{\mathrm{R}_{-} \text {Min }}$ does not fall as low in the proposed scheme as it does in the existing scheme. However, as $P_{\mathrm{R} \text { Min }}$ shoots widely in the existing scheme, it may often rise above the proposed scheme as occurs around user speed $140 \mathrm{~km} / \mathrm{hr}$. Thus, in the existing scheme, $P_{\mathrm{R} \_ \text {Min }}$ may get better, although rarely, but nevertheless, its instability remains as a problem. Figure 4 also shows that a decrease in SSSF triggers cell reselections earlier boosting the level 
of $P_{\mathrm{R} \text { Min }}$ and thus, it indicates how the UE can quickly perform necessary adjustment using SSSF.

Figure 5 demonstrates the actual proposal of establishment of a set point using a fixed $Q_{\text {hyst }} S F$ value and the use of instant adjustment using $S S S F$ as an overlay. In Fig. 5, several fixed $Q_{\text {hyst }} S F$ values are used to establish different set points. On top of each of them, the UE gradually increases $S S S F$ values as the user speed increases. It is evident from Fig. 5 that the profile of $P_{\mathrm{R} \_ \text {Min }}$ is under good control using $Q_{\text {hyst }} S F$ and $S S S F$, selected by the eNodeB and the UE, respectively. Using an appropriate control, $P_{\mathrm{R} \text { Min }}$ values should be made as velocity independent as seems justified. The proposed scheme can achieve good stability of $P_{\mathrm{R}_{-} \text {Min }}$. The eNodeB and the UE, from the two ends, can employ the enhanced controllability considering the requirements of various factors of the particular scenario according to the guidelines shown in Sec. 5.3. Thus, the triggering of cell reselection will potentially adapt to various scenarios in a desired fashion. It may be noted that an attempt to raise $P_{\mathrm{R} \_ \text {Min }}$ too high using $Q_{\mathrm{hys}} S F$ and $S S S F$ may lead to ping-pong effects. The user moves along Trajectory 2 in Fig. 6 . The results of Fig. 4 and Fig. 6 are found to be very similar. Figure 6 basically shows that the proposed scheme is helpful in a different pathway too.

\subsection{Results for Handover}

Figures 7 to 9 show $P_{\mathrm{R} \_ \text {Min }}$ vs. user speed profile comparing existing and proposed schemes in the case of handover with a fixed value of Hyst_SF and different values of SSSF. The hysteresis is not scaled in the existing scheme as mentioned in Sec. 3.3 but it is scaled in the proposed scheme. To show the impact of this scaling, the proposed scheme avoids scaling of hysteresis in Fig. 7 whereas this scaling is applied in all other figures in this section. In Fig. 7, $P_{R_{-} \text {Min }}$ is found significantly higher in the proposed scheme compared to the existing scheme but its stability with the user speed is not a lot better. With scaling of hysteresis, in Fig. 8, $P_{\mathrm{R}_{-} \text {Min }}$ stays far higher in the proposed scheme compared to the existing scheme and also, the stability of $P_{\mathrm{R}_{-} \text {in }}$ is way better in the proposed scheme. The decrease in SSSF triggers handover earlier boosting the level of $P_{\mathrm{R} \text { Min }}$ and thus, it indicates how the UE can quickly perform necessary adjustment using $S S S F$.

In the existing scheme, the received power may remain very low for a period and in this period, the service is extremely degraded and it is at risk of disruption. Therefore, the length of this period is determined assuming that the service degrades extremely when the received power falls below $-90 \mathrm{dBm}$. Table 2 shows the length of the period with such low received power. This period is not calculated for the proposed scheme as the received power is then controlled to stay above $-90 \mathrm{dBm}$. Table 2 shows that the periods are not trivial. The period increases gradually with the user speed because the user goes further away from the old serving cell before handover. However, the period decreases between user speed $90 \mathrm{~km} / \mathrm{hr}$ and $110 \mathrm{~km} / \mathrm{hr}$ because of a change in mobility state.

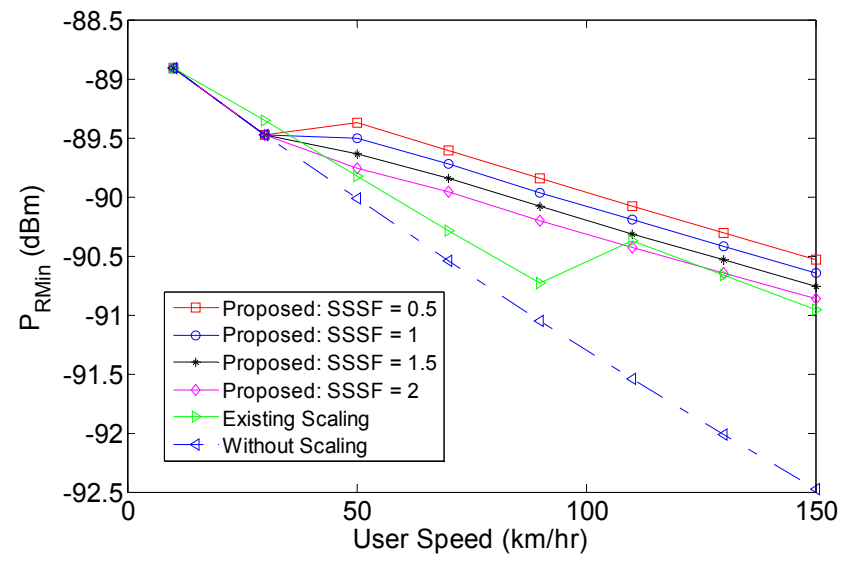

Fig. 7. $P_{\mathrm{R} \_ \text {Min }}$ vs. user speed for $\mathrm{HO}$ avoiding scaling of hysteresis in the proposed scheme (Hyst_SF=-0.4 dB).

\begin{tabular}{|c|c|c|c|c|c|}
\hline $\begin{array}{c}\text { Velocity } \\
\text { (km/hr) }\end{array}$ & 70 & 90 & 110 & 130 & 150 \\
\hline $\begin{array}{c}\text { Period } \\
\text { (ms) }\end{array}$ & 696 & 1404 & 574 & 886 & 1114 \\
\hline
\end{tabular}

Tab. 2. Period of power reception below $-90 \mathrm{dBm}$ in the existing scheme.

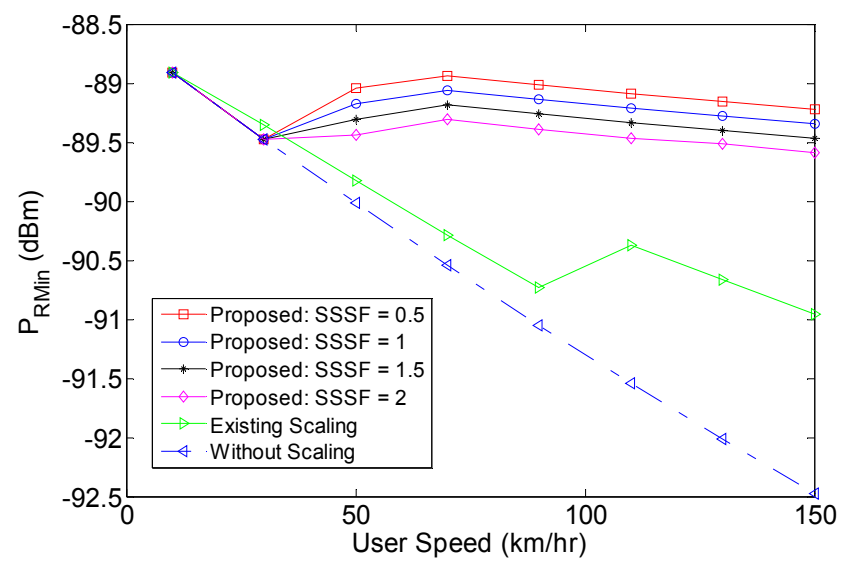

Fig. 8. $\quad P_{\mathrm{R}_{\_} \text {Min }}$ vs. user speed for $\mathrm{HO}$ with scaling of hysteresis in the proposed scheme (Hyst_SF $=-0.4 \mathrm{~dB}$ ).

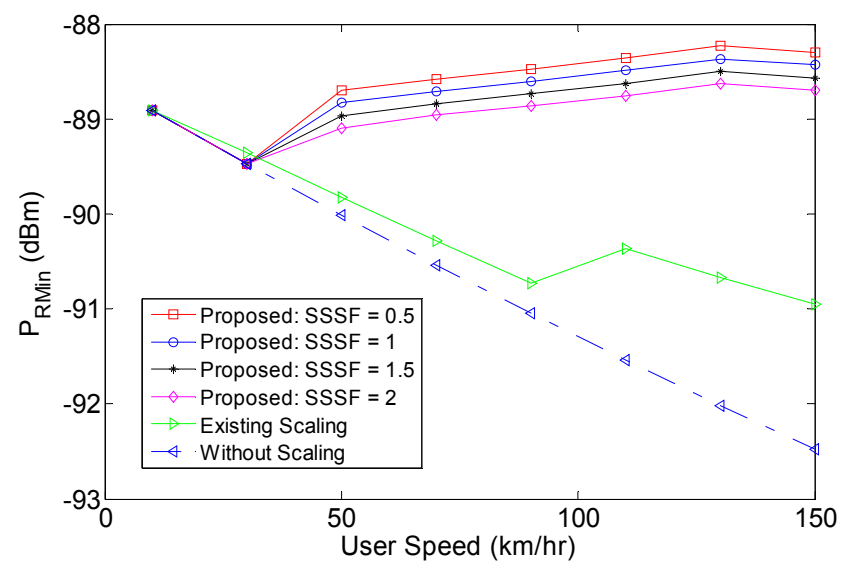

Fig. 9. $P_{\mathrm{R}_{\_} \text {Min }}$ vs. user speed for $\mathrm{HO}$ with scaling of hysteresis in the proposed scheme (Hyst_SF $=-0.7 \mathrm{~dB}$ ).

In Figure 9, the absolute value of Hyst_SF is higher compared to Fig. 8 and thus, Figure 9 has higher $P_{\mathrm{R}_{\_} \text {Min }}$ values for the same SSSF values. This shows that, in the 


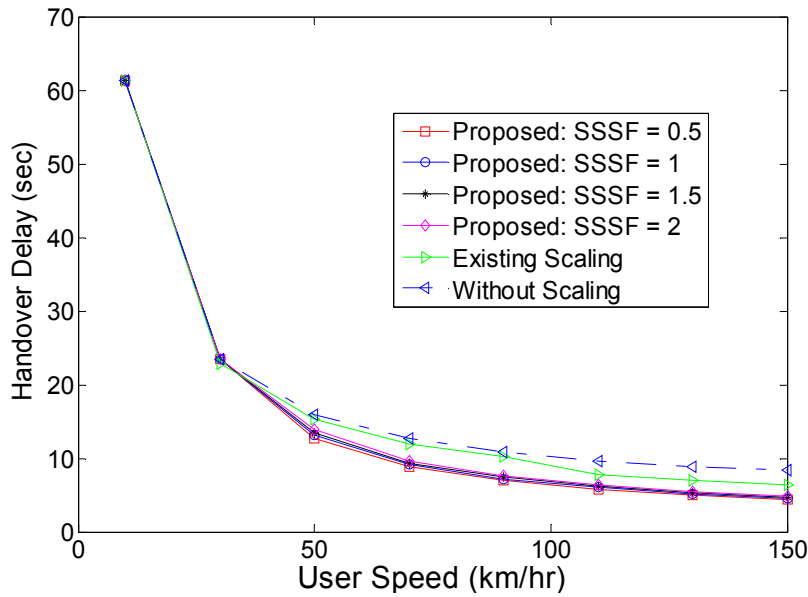

Fig. 10. Handover delay vs. user speed for $\mathrm{HO}$ with scaling of hysteresis in the proposed method (Hyst_SF $=-0.4 \mathrm{~dB})$.

proposed scheme, the eNodeB and the UE, from the two ends, can apply good control using Hyst_SF and SSSF, respectively. To ensure that the triggering of handover adapts to various scenarios in the desired fashion, the enhanced controllability should consider various factors, for example, user speeds, traffic loads, street patterns, types of cells, and so forth, according to the guidelines shown in Sec. 5.3.

Figure 10 shows the handover delay vs. user speed. The delay is calculated as the time that elapses between the moment of crossing the cell boundary and the moment of triggering handover. The lowest value of the delay is $4417 \mathrm{msec}$ in the proposed scheme and it occurs at user velocity $150 \mathrm{~km} / \mathrm{hr}$ with $S S S F$ 0.5. In a measurement period of $200 \mathrm{msec}$, the physical layer of the UE performs measurements of at least 8 identified intra-frequency cells and reports the results to layer 3 when no measurement gaps are activated [25]. Thus, in $4417 \mathrm{msec}$, assuming the presence of 16 intra-frequency cells, the layer 3 of the UE receives measurement results at least 11 times from the physical layer until it sends its last measurement report. This appears to be sufficient to ensure that the neighbor cell has really got better than the serving cell avoiding the possibility of ping-pong effects.

\section{Conclusion}

The challenges in the mobility support for speedy users in HetNets have been investigated and a wide variety of issues are derived. Addressing most of the issues, a scheme is proposed with versatile controllability over the scaling of cell switching parameters. Some of the features of the proposed scheme have been evaluated using limited simulations. The simulation results show that the lowest received power improves and also, it stabilizes better with the user speed in the proposed scheme. The illustrations of the proposed scheme bespeak the potential mitigation of various problems and the achievement of satisfactory cell edge performance.

\section{References}

[1] KAWSER, M. T. LTE Air Interface Protocols. Boston (USA): Artech House, 2011. ISBN: 978-1-60807-201-9

[2] Fujitsu Network Communications Inc. Enhancing LTE Cell-Edge Performance via PDCCH ICIC. 16 pages. [Online] Cited 2011. Available at: http://www.fujitsu.com/us/Images/Enhancing-LTECell-Edge.pdf

[3] WILEY-GREEN, M. P., SVENSSON, T. Throughput, capacity, handover and latency performance in a 3GPP LTE FDD field trial. In Proceedings of IEEE Global Telecommunications Conference (GLOBECOM 2010). Florida (USA), December 2010, p. 1-6. DOI: $10.1109 /$ GLOCOM.2010.5683398

[4] 3GPP TR 36.839. Evolved Universal Terrestrial Radio Access (EUTRA); Mobility Enhancements in Heterogeneous Networks. Release 11, 2012.

[5] 3GPP TS 36.331. Evolved Universal Terrestrial Radio Access (EUTRA); Radio Resource Control (RRC); Protocol specification. Release 11, 2014.

[6] LOPEZ-PEREZ, D., GUVENC, I., CHU, X. Mobility enhancements for heterogeneous wireless networks through interference coordination. In Proceedings of IEEE Wireless Communications and Networking Conference Workshops $(W C N C W)$. Paris (France), April 2012, p. 69-74. DOI: 10.1109/WCNCW.2012.6215543

[7] SIMSEK, M., BENNIS, M., GUVENC, I. Mobility management in HetNets: a learning-based perspective. EURASIP Journal on Wireless Communications and Networking, February 2015, vol. 14. DOI 10.1186/s13638-015-0244-2

[8] PENG, Y., YANG, Y.Z.W., ZHU, Y. Mobility Performance enhancements for LTE-Advanced heterogeneous networks. In Proceedings of IEEE $23^{\text {rd }}$ International Symposium on Personal Indoor and Mobile Radio Communications (PIMRC). Sydney (Australia), September 2012, p. 413-418. DOI: 10.1109/PIMRC.2012.6362820

[9] BARBERA, S., MICHAELSEN, P., SAILY, M., PEDERSEN, K. Mobility performance of LTE co-channel deployment of macro and pico cells. In Proceedings of IEEE Wireless Communications and Networking Conference (WCNC). Paris (France), April 2012, p. 2863-2868. DOI: 10.1109/WCNC.2012.6214290

[10] BARBERA, S., MICHAELSEN, P., SAILY, M., PEDERSEN, K. Improved mobility performance in LTE co-channel HetNets through speed differentiated enhancements. In Proceedings of IEEE Globecom Workshops (GC Wkshps). Anaheim (CA, USA), Dec. 2012, p. 426-430. DOI: 10.1109/GLOCOMW.2012.6477610

[11] ZHANG, H., WEN, X., WANG, B., ZHENG, W., SUN, Y. A novel handover mechanism between femtocell and macrocell for LTE based networks. In Proceedings of IEEE Second International Conference on Communication Software and Networks (IC$C S N)$. February 2010, p. 228-231. DOI: 10.1109/ICCSN.2010.91

[12] PEDERSEN, K.I., MICHAELSEN, P.H., ROSA, C., BARBERA, S. Mobility enhancements for LTE-advanced multilayer networks with inter-site carrier aggregation. IEEE Communications Magazine, May 2013, vol. 51, no. 5, p. 64-71. DOI: 10.1109/MCOM.2013.6515048

[13] PENG, Y., YANG, W., ZHANG, Y., ZHU, Y. Mobility performance enhancements for LTE-advanced heterogeneous networks. In Proceedings of IEEE $23^{\text {rd }}$ International Symposium on Personal Indoor and Mobile Radio Communications (PIMRC). Sydney (Australia), September 2012, p. 413-418. DOI: 10.1109/PIMRC.2012.6362820

[14] LEE, Y., SHIN, B., LIM, J., HONG, D. Effects of time-to-trigger parameter on handover performance in SON-based LTE systems. 
In Proceedings of IEEE $16^{\text {th }}$ Asia-Pacific Conference on Communications (APCC). Auckland (New Zealand), OctoberNovember 2010, p. 492-496. DOI: 10.1109/APCC.2010.5680001

[15] JANSEN, T., BALAN, I., TURK, J., MOERMAN, I., KURNER, T. Handover parameter optimization in LTE self-organizing networks. In Proceedings of IEEE $72^{\text {nd }}$ Vehicular Technology Conference Fall (VTC 2010-Fall). Ottawa (Canada), September 2010, p. 1-5. DOI: 10.1109/VETECF.2010.5594245

[16] ZHENG, W., ZHANG, H., CHU, X., WEN, X. Mobility robustness optimization in self-organizing LTE femtocell networks. EURASIP Journal on Wireless Communications and Networking, 2013, 10 p. DOI: 10.1186/1687-1499-2013-27

[17] EMRAH TUNÇEL. Tuning of Handover Parameters in LTE-A Heterogeneous Networks. 94 pages. [Online] Cited 2014-09. Available at: http://etd.lib.metu.edu.tr/upload/12617911/index.pdf

[18] KIM, Y., LEE, K., CHIN, Y. Analysis of multi-level threshold handoff algorithm. In Proceedings of IEEE Global Telecommunications Conference (GLOBECOM 96). London (UK), November 1996, p. 1141-1145. DOI: 10.1109/GLOCOM.1996.587613

[19] KAWSER, M. T., ISLAM, M. R., AHMED, K. I., et al. Efficient resource allocation and sectorization for fractional frequency reuse (FFR) in LTE femtocell systems. Radioengineering, December 2015, vol. 24, no. 4, p. 940-947. DOI: 10.13164/re.2015.0940

[20] AAMOD KHANDEKAR, NAGA BHUSHAN, JI TINGFANG, et al. LTE Advanced: Heterogeneous networks. In Proceedings of IEEE European Wireless Conference (EW). Lucca (Italy), April 2010, p. 978-982. DOI: 10.1109/EW.2010.5483516

[21] SCHOBER, H., JONDRAL, F. Velocity estimation for OFDM based communication systems. In Proceedings of IEEE $56^{\text {th }}$ Vehicular Technology Conference (VTC 2002-Fall). Vancouver, (BC, Canada), September 2002, vol. 2, p. 715-718. DOI: 10.1109/VETECF.2002.1040692

[22] YUCEK, T., TANNIOUS, R. M. A., ARSLAN, H. Doppler spread estimation for wireless OFDM systems. In Proceedings of IEEE/Sarnoff Symposium on Advances in Wired and Wireless Communication. Princeton (NJ, USA), April 2005, p. 233-236. DOI: $10.1109 /$ SARNOF.2005.1426552

[23] TEPEDELENLIOGLU, C., ABDI, A., GIANNAKIS, G. B., et al. Estimation of Doppler spread and signal strength in mobile communications with applications to handoff and adaptive transmission. Wireless Communications and Mobile Computing, April 2001, vol. 1, no. 2, p. 221-242. DOI: 10.1002/wcm.1

[24] BHATTACHARYA, P. P. A new environment dependent handoff technique for next generation mobile systems. International
Journal of Computer and Communications, March 2011, vol. 1, no. 1, p. $15-24$.

[25] 3GPP TS 36.133 V11.4.0. Evolved Universal Terrestrial Radio Access (E-UTRA); Requirements for Support of Radio Resource Management. Release 12, 2014.

\section{About the Authors ...}

Mohammad T. KAWSER received his Ph.D. degree from Islamic University of Technology, Bangladesh in 2016, MS degree from Virginia Tech, USA in 2005 and BS degree from Bangladesh University of Engineering and Technology, Bangladesh in 1999, all in Electrical and Electronic Engineering. He is serving as an assistant professor at Islamic University of Technology, Bangladesh. His research interests include layer 2 and layer 3 functions of cellular operation, LTE-Advanced features, etc.

Mohammad R. ISLAM received BS and MS degree in Electrical and Electronic Engineering from Bangladesh University of Engineering and Technology, Bangladesh in 1998 and 2004, respectively. He received MBA degree from the Institute of Business Administration, Bangladesh in 2006. He received his Ph.D. degree from Kyung Hee University, South Korea in 2010. He is serving as a professor at Islamic University of Technology, Bangladesh. His research interests include wireless sensor networks, LDPC and QC-LDPC codes, secrecy capacity and other wireless applications.

Muhammad R. RAHIM received his BS degree in Electrical and Electronic Engineering from Islamic University of Technology in 2012. Currently, he is enrolled in the MS program at RWTH Aachen University, Germany. His interest lies in mobile radio networking.

Muhammad A. MASUD received his BS degree in Electrical and Electronic Engineering from Islamic University of Technology in 2012. He has been serving as a lecturer at Uttara University, Bangladesh. His research interests include wireless communications, inter-networking and network security. 